\title{
KV Encontro Nacional de Tecnologia do Ambiente Construído
}

A RELAÇÃO ENTRE A COMPACIDADE DE PRÉDIOS E MONTANTE DE ENERGIA INCORPORADA E EMISSÕES DE $\mathrm{CO}_{2}$ EM EHIS

\author{
POSTAY, Renata (1); KERN, Andrea P. (2); MANCIO, Maurício (3); \\ GONZÁLEZ, Marco A. S. (4); SCHNECK, Eduardo R. (5); HEHN, Alan V. (6)
}

(1) UNISINOS, e-mail: rpostay@gmail.com, (2) UNISINOS, e-mail: apkern@unisinos.br, (3) UNISINOS, e-mail: mancio@unisinos.br, (4) UNISINOS, e-mail: mgonzález@unisinos.br, (5) FEEVALE, e-mail: eduardoschneck@gmail.com, (6) UNISINOS, e-mail: alanhehn@gmail.com.

\begin{abstract}
RESUMO
A construção civil é considerada como uma das atividades que consome uma grande quantidade de energia. Destaca-se o setor residencial, que utiliza o equivalente aos setores comercial e público juntos, em todas as fontes de energia, envolvendo desde a energia para a produção de materiais e componentes, como a energia utilizada na fase de uso do ambiente construído. Práticas de ações voltadas à economia e da construção, desde a fase de projeto, até a execução são muito oportunas, tendo em vista a atual crise energética que o país vem enfrentando. Esse artigo investiga a relação entre compacidade de prédios de empreendimentos de habitação de interesse social (EHIS) com o montante de energia incorporada (EI) e emissões de dióxido de carbono $\left(\mathrm{CO}_{2}\right)$ dos materiais de construção. Entendendo EI como o montante energético utilizado para a produção de determinado produto e ponderando o $\mathrm{CO}_{2}$ como um dos principais gases colaboradores do efeito estufa. Ao todo foram calculados os montantes de EI de cinco prédios de EHIS, considerando áreas similares e o mesmo sistema construtivo de alvenaria estrutural de blocos cerâmicos. A variável do estudo consistiu no índice econômico de compacidade dos prédios, que mudou conforme a configuração geométrica dos mesmos. Os resultados mostram que quanto maior a compacidade dos prédios, menor a quantidade de EI e emissões de $\mathrm{CO}_{2}$, chegando a diferença de $20 \%$ para EI e $21 \%$ para emissões de $\mathrm{CO}_{2}$ entre os 5 projetos analisados.
\end{abstract}

Palavras-chave: Projeto, Empreendimento habitacional de interesse social, Índice econômico de compacidade, Energia Incorporada, Emissões de $\mathrm{CO}_{2}$.

\begin{abstract}
The civil construction is considered as one of the activities that consume a lot of energy. The residential sector is highlighted because it uses the energy equivalent of the commercial and public sectors together, considering all energy sources, such as the energy to produce materials and components, and also the energy used in the use phase of the built environment. Practices of actions aimed to the economy of construction, from the design phase to execution are very timely, given the current energy crisis that the country is facing. This paper investigates the relationship between compactness of buildings from ventures of social interest housing, with the amount of embodied energy (EE) and dioxide carbon emissions $\left(\mathrm{CO}_{2}\right)$ of building materials. Understanding EE as the amount of energy used for the production of certain product and considering $\mathrm{CO}_{2}$ as a leading contributor of greenhouse gases. Five buildings had the amount of EE calculated, considering similar areas and the same constructive system of structural masonry with ceramic blocks. The variable of the study consisted on the economic index compactness of the buildings, which changed according to the geometrical configuration of the same. The results show that the higher is the compactness of the buildings, the lower is the amount of EE and $\mathrm{CO}_{2}$ emissions, reaching the divergence from $20 \%$ to $21 \%$ for $\mathrm{EE}$ and $\mathrm{CO}_{2}$ emissions, between the five projects analyzed.
\end{abstract}

Keywords: Design, Social housing design, Economic index of compactness, Embodied energy, $\mathrm{CO}_{2}$ Emissions. 


\section{INTRODUÇÃO}

A matriz energética brasileira possui $45,3 \%$ da produção proveniente de fontes como recursos hídricos, biomassa e etanol, além das energias eólica e solar, sendo as usinas hidrelétricas responsáveis pela geração de mais de $75 \%$ da eletricidade do País (BRASIL, 2014). Contudo, embora a matriz energética seja de fonte renovável e limpa, é recorrente a preocupação em reduzir o consumo de energia do Brasil (PAULSEN; SPOSTO, 2013). Períodos de falta de chuva aliados ao aumento no consumo de energia e falta de investimentos no setor tem sido apontados como causas dos recentes apagões ocorrido nas diferentes regiões do país. A crise energética se tornou assim, um tema de suma importância e urgência, com presença marcante na pauta atual de discussão no país.

Tanto a indústria da construção como o ambiente construído são considerados duas áreas chave para o desenvolvimento sustentável na sociedade e apresentam papel essencial para o desenvolvimento de tecnologias mais limpas e enxutas (HALLIDAY, 1997 apud LOBO et al., 2010). Na lista das 10 atividades consideradas maiores consumidoras de energia do país estão seis da construção civil (LOBO, SANTOS, TAVARES; 2010). Entre os setores diretamente ligados à construção civil, destaca-se a importância do setor residencial em termos energéticos, por consumir o equivalente aos setores comercial e público juntos, em todas as fontes de energia (BRASIL, 2005).

Esse maciço consumo energético da construção civil, cria oportunidades para se pensar em gestão e planejamento para avaliação ambiental, envolvendo desde a energia para a produção de materiais e componentes, como a energia utilizada na fase de uso do ambiente construído. A prática de ações voltadas à desmaterialização da economia e da construção, que envolvem medidas eficientes por parte dos profissionais desde a fase de planejamento, projeto, até a execução. Ou seja, construir mais usando menos materiais, reduzindo a pressão sobre a natureza e o volume de material nos aterros, sem aumentar outros impactos ambientais (WEINSTOCK, 2000; AGOPYAN e JOHN, 2011).

O projeto é um dos elementos fundamentais do processo de produção no setor da construção, sendo o elemento indutor da racionalização da construção, da qualidade do produto final e de sua sustentabilidade, pois é na fase de projeto que o produto é concebido e os materiais e as técnicas construtivas são especificados (CARVALHO; SPOSTO, 2012). Como resultado da escolha dos materiais e forma definida tem-se o impacto ambiental, podendo citar: o consumo de materiais, energia incorporada, emissões de $\mathrm{CO}_{2}$ (SCHNECK, 2013) e geração de resíduos (DIAS, 2013).

Nos últimos anos o segmento de habitação de interesse social tem recebido grandes investimentos do Governo Federal, na busca para redução do alto déficit habitacional que o país enfrenta há décadas. De acordo com dados disponibilizados pela CEF (2011) entre os anos de 2003 até dezembro de 2010 foi realizada a contratação de um milhão e cinco mil unidades habitacionais no âmbito do Programa Minha Casa Minha Vida (PMCMV), sendo 936,5 mil pela Caixa Federal, com estimativa de chegar até o final de 2014 a 2 milhões de casas e apartamentos construídos.

Brandão (2006) aponta a necessidade de mais pesquisas focadas na fase de projeto de EHIS, considerando que parte das plantas consideradas típicas nesse segmento não configuram arranjos com os melhores índices de conforto, além de apresentarem baixo nível de construtibilidade e qualidade ao usuário. Heineck e Fernandes (2004) salientam que a análise de projetos pode ser direcionada a diversos aspectos de ordem qualitativa: a estética, a geometria funcional, a geometria morfológica, a construtibilidade e a racionalidade. Esses aspectos, perante as decisões tomadas na fase de projeto, podem 
ser correlacionados a impactos ambientais (SCHNECK, 2013). Assim emerge uma demanda por traçar um paralelo entre a eficiência geométrica dos arranjos físicos propostos com os aspectos ambientais envolvidos enquanto os EHIS estão sendo concebidos.

Tratando-se de habitação de interesse social, os parâmetros dimensionais são limitados pela relação direta que estabelecem com o custo da construção, um importante requisito neste segmento. Segundo Mayer (2012), mantendo-se constante o tipo e qualidade do material empregado, uma das formas de parametrizar o custo da construção está diretamente relacionado com a sua compacidade: quanto mais compacta a construção, menor o seu custo. Onde, a diminuição do custo se dá pela menor necessidade de materiais.

A compacidade do prédio pode ser representado pelo índice de compacidade definido, por Rosso (1978), pela relação percentual que se estabelece entre o perímetro de um círculo de igual área do projeto e o perímetro de paredes exteriores do projeto (Equação 1), definido na década de 70, pelo Building Performance Research Unit da Universidade escocesa de Strathclyde.

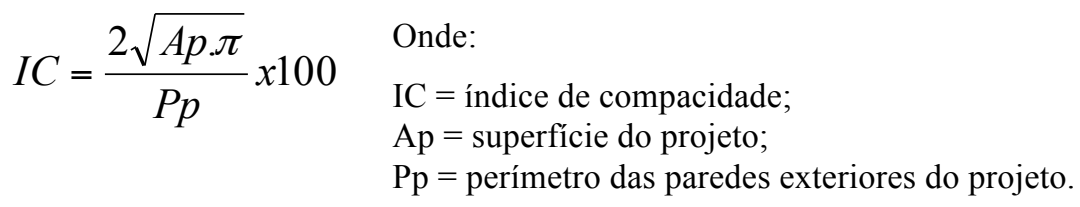

A partir de uma revisão feita por Mascaró (2010), o índice teve incorporado o número de arestas e perímetros curvos de fachadas, em função do maior custo de execução destes, passando à denominação atual de Índice Econômico de Compacidade (IeC), conforme Equação 2.

$$
\begin{aligned}
& I e C=\frac{2 \sqrt{A p \cdot \pi}}{P e p} x 100 \quad \begin{array}{l}
\text { Onde: } \\
\mathrm{IeC}=\text { índice econômico de compacidade; }
\end{array} \\
& \mathrm{Ap}=\text { superfície do projeto; } \\
& \text { Pep }=\text { perímetro econômico do projeto. }
\end{aligned}
$$

O valor do Pep da Equação 2 é calculado separadamente pela fórmula da Equação 3, sendo que é este valor que difere o IeC do IC, devido a ponderar arestas e curvas no perímetro.

$$
\begin{aligned}
P e p=P p r+1,5 P p c+\frac{n A}{2} \quad & \text { Onde: } \\
& \mathrm{Pep}=\text { perímetro econômico do projeto; } \\
& \mathrm{Ppr}=\text { perímetro das paredes exteriores retas; } \\
& \mathrm{Ppc}=\text { perímetro das paredes exteriores curvas; } \\
& \mathrm{nA}=\text { número de arestas das fachadas. }
\end{aligned}
$$

O objetivo deste artigo é investigar a relação entre compacidade de prédios de EHIS com o montante de energia incorporada (EI) e emissões de $\mathrm{CO}_{2}$ dos materiais de construção. Entendendo EI como o montante energético utilizado para a produção de determinado produto, desde a extração, distribuição no mercado até sua destinação final (GAUZIN-MÜLLER, 2002) e ponderando-se $\mathrm{o} \mathrm{CO}_{2}$ como um dos principais gases colaboradores do efeito estufa.

Esta artigo apresenta resultados parciais de uma Dissertação de Mestrado que está em desenvolvimento no âmbito de um projeto de pesquisa realizado com fomento do 
MCTI/CNPq/MCidades, Chamada 11/2012, e tem como título: "Projetos de Empreendimentos de Habitação de Interesse Social: conjunto de indicadores para avaliação do desempenho urbano, ambiental e quesitos de habitabilidade".

\section{MÉTODO DE PESQUISA}

O método empregado no estudo utilizou como estratégia de pesquisa os estudos de caso, tendo como objeto de estudo: projetos arquitetônicos de EHIS construídos no Rio de Janeiro e São Leopoldo, além de um caso proposto por Schneck (2013). O critério de escolha dos projetos foram programas, áreas e número de pavimentos semelhantes, considerando para todos o mesmo sistema construtivo, de alvenaria estrutural com blocos cerâmicos. Assim, a variável dependente do trabalho foi a compacidade dos prédios, buscando-se configurações geométrica distintas. As etapas da execução encontram-se sintetizada no delineamento da pesquisa demonstrado na Figura 1.

\section{Figura 1 - Delineamento da Pesquisa}

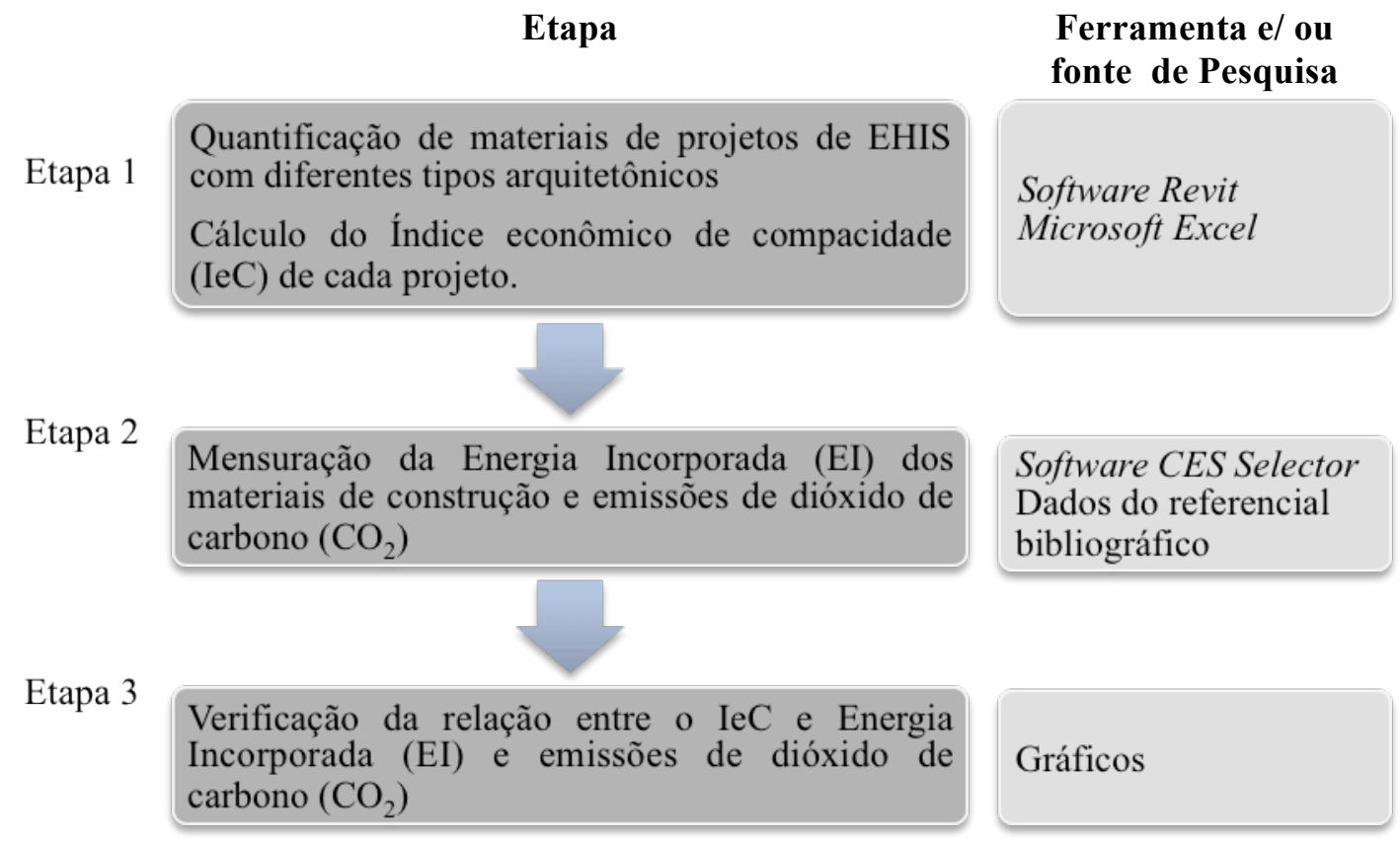

O desenvolvimento da pesquisa iniciou (etapa 1) nos estudos de caso, ao todo foram analisados projetos arquitetônicos de cinco EHIS, sendo que quatro deles pertencem ao Programa Minha Casa Minha Vida. Três são empreendimentos construídos na cidade do Rio de Janeiro - RJ (no estudo denominados P2, P3 e P4) e um situado na cidade de São Leopoldo - RS (denominado P1). Já o último pertence ao estudo de caso utilizado por Schneck (2013) e consiste numa simulação com base no P1, projeto localizado em São Leopoldo, onde o tipo arquitetônico do prédio foi alterado a fim de aumentar o IeC. Esse projeto é utilizado como base de comparação nos estudos, sendo denominado P5.

O Quadro 1 apresenta as características dos projetos analisados nessa pesquisa. 


\section{Quadro 1 - Síntese dos projetos}

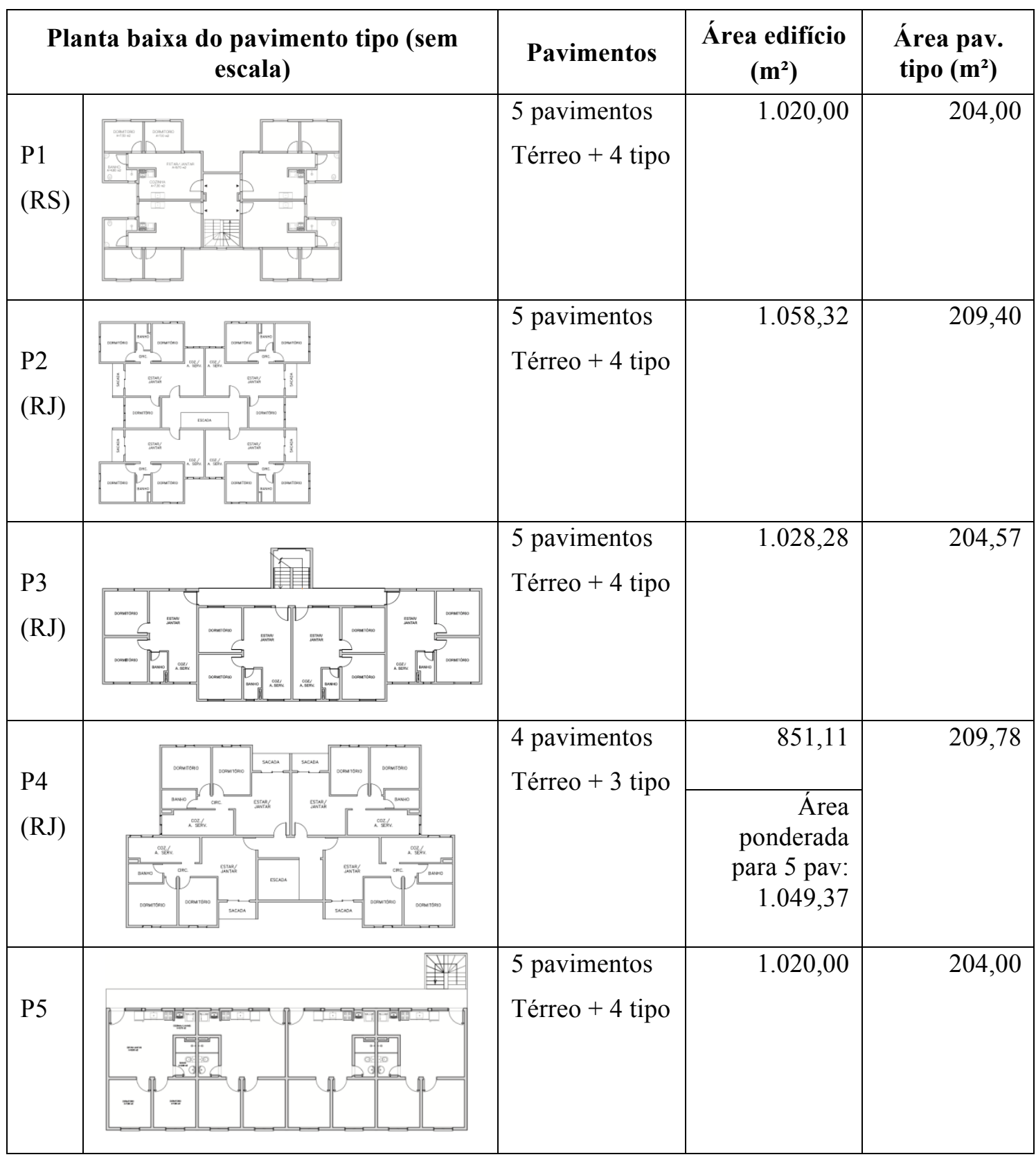

A partir das características dos cinco empreendimentos analisados observa-se que os cinco possuem 4 apartamentos por pavimento, tanto no térreo como no pavimento tipo. Quatro dos cinco projetos possuem 5 pavimentos, exceto o P4 que possui apenas 4 pavimentos, vale destacar que o P4 para parâmetros de comparação de quantitativos de materiais e emissões foi considerado como 5 pavimentos (área $1.049,37 \mathrm{~m}^{2}$ ). Importante salientar também que no P2 e P4 os apartamentos possuem sacadas privativas, que estão contempladas no computo da área total do pavimento. Já quanto a área do pavimento tipo, há variação entre $204,00 \mathrm{~m}^{2}$ e $209,78 \mathrm{~m}^{2}$, o que significa um intervalo de $5,78 \mathrm{~m}^{2}$.

A primeira atividade realizada consistiu em quantificar o consumo de materiais necessários para a construção e calcular o índice econômico de compacidade (IeC) de cada um dos cinco projetos. Onde, os projetos dos empreendimentos, disponibilizados pela Caixa Econômica Federal e o projeto simulado por Schneck (2013), foram 
modelados no software Revit Autodesk, programa com a tecnologia BIM (Building Information Modeling).

A partir dos dados do programa, foi gerada uma lista de materiais e seus respectivos quantitativos (em quilogramas), estruturada conforme as etapas de obra, baseada na estrutura do quantitativo do P1, tendo em vista que no estudo de Schneck (2013) o quantitativo foi a partir do empresa construtora do projeto. Sendo ponderadas algumas considerações sobre os materiais: (a) para alguns materiais que não se obteve as quantidades no Revit, foram extrapoladas comparações com quantitativo de P1; (b) para os serviços preliminares foram considerados os mesmos valores para os 5 projetos, tendo como base $\mathrm{P} 1$; (c) foram excluídos do cômputo os materiais referentes a esquadrias, instalações elétricas, instalações hidráulicas e pinturas. Posterior a isso, os materiais iguais foram agrupados para facilitar a inserção deles no programa a ser utilizado na etapa subsequente.

A partir do quantitativo dos materiais, parte-se para a Etapa 2, que consiste na análise dos impactos ambientais dos 5 projetos, quanto à energia incorporada e emissões de $\mathrm{CO}_{2}$, em função dos materiais empregados e respectivas quantidades. Atividade realizada com auxílio do software CES Selector, que viabiliza uma análise detalhada dos impactos ambientais de produtos ao longo do seu ciclo de vida, com a ferramenta Eco Audit Tool, onde foram selecionados os materiais na base de dados do programa e inseridas as quantidades de cada um deles. Para os materiais que não constavam na base de dados do programa, foi utilizado o módulo Constructor, que permite a personalização da base de dados, possibilitando inserir características próprias dos estudos analisados e especificações locais, para posterior inclusão no Eco Audit Tool. Para suporte a esses materiais da nova base de dados, foram considerados também como base os valores de EI e emissão de $\mathrm{CO}_{2}$ dos materiais de construção das pesquisas de Tavares (2006) e Lobo, Santos e Tavares (2010).

Após a obtenção dos dados de consumo de EI e emissões de $\mathrm{CO}_{2}$ dos projetos em estudo, na Etapa 3, foram montados gráficos para comparar os resultando, sendo relacionado o IeC resultante das decisões de projeto quanto ao tipo arquitetônico, ao consumo de EI e emissões de $\mathrm{CO}_{2}$.

\section{RESULTADOS E DISCUSSÕES}

Os IeC de cada um dos cinco projetos são apresentados no Quadro 2, calculados a partir da Equação 2, onde apresentam variação de 49,40 (P1) até o maior índice de 72,12 (P5).

\section{Quadro 2 - IeC dos Projetos}

\begin{tabular}{|c|c|c|c|c|c|}
\hline & P 1 & P 2 & P 3 & P 4 & P 5 \\
\hline IeC & 49,40 & 54,44 & 62,71 & 64,21 & 72,12 \\
\hline
\end{tabular}

Quanto a energia incorporada e emissões de $\mathrm{CO}_{2}$ temos os gráficos apresentados respectivamente na Figura 2 e Figura 3, onde estão apresentados de ordem decrescente de energia incorporada/ emissão de $\mathrm{CO}_{2}$, gráfico gerado pelo software CES Selector. 
Figura 2 - Estimativa de EI dos cinco projetos

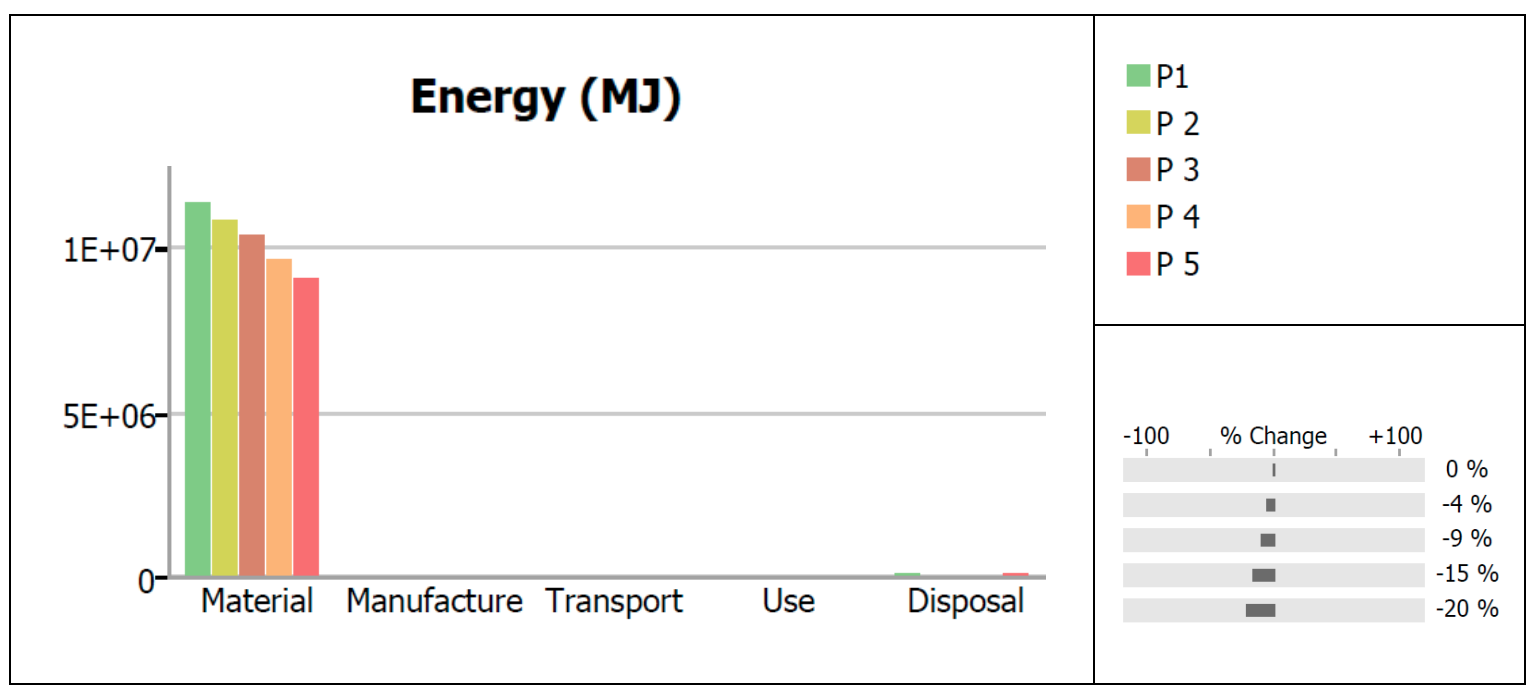

Figura 3 - Estimativa de emissões de $\mathrm{CO}_{2}$ dos cinco projetos

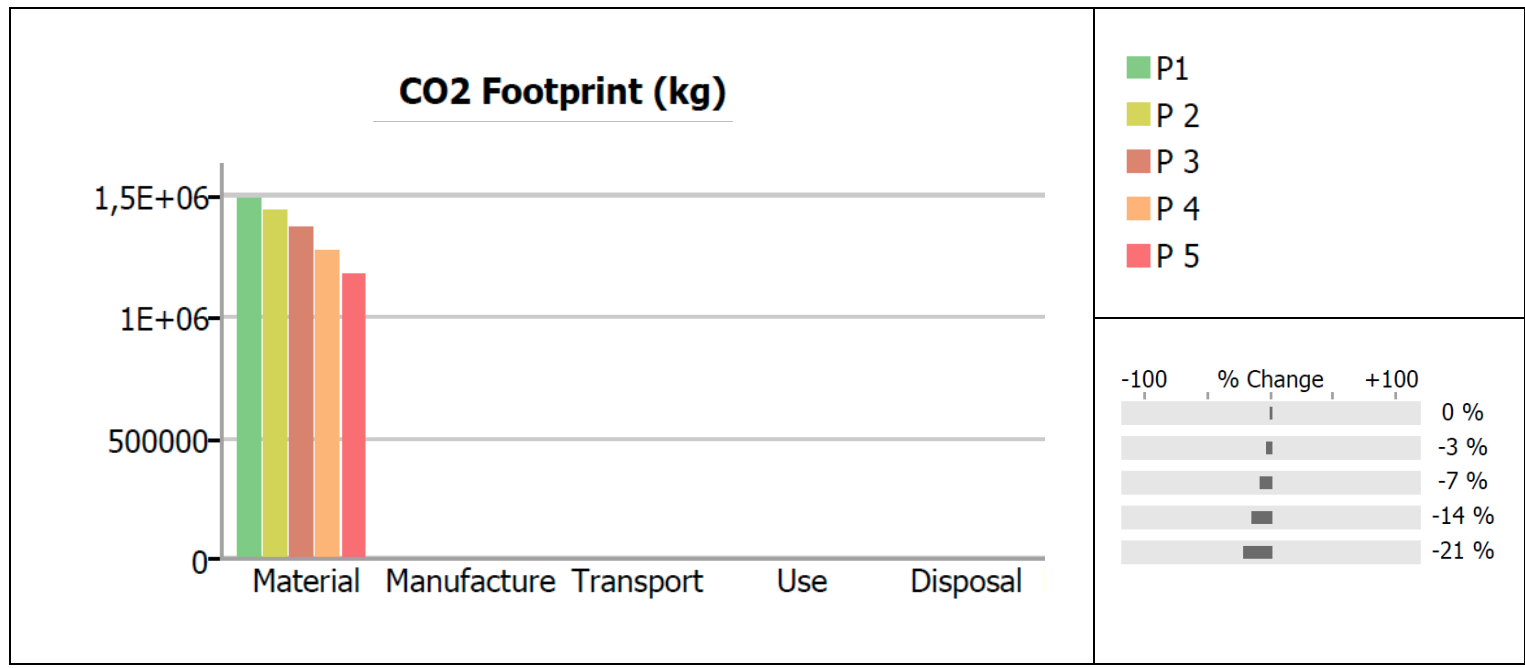

Tanto para estimativa de EI quanto emissões de $\mathrm{CO}_{2}$ o projeto com maior impacto ambiental foi o P1 considerando os aspectos analisados, sendo utilizado como parâmetro de comparação para os demais projetos. Assim, a partir dele tem-se a redução quanto a EI de 4\% para $\mathrm{P} 2,9 \%$ de redução para $\mathrm{P} 3,15 \%$ para $\mathrm{P} 4$ e $20 \%$ para P5 e quanto as emissões de $\mathrm{CO} 2$, ao comparar os projetos com P4 tem-se: redução de $3 \%$ para $\mathrm{P} 2,7 \%$ para $\mathrm{P} 3,14 \%$ para $\mathrm{P} 4$ e $21 \%$ para $\mathrm{P} 5$.

Traçando-se um paralelo entre os dados de EI e IeC, como se observa na Figura 4, e também entre emissões de $\mathrm{CO}_{2}$ e IeC, demonstrado na Figura 5, quanto maior o IeC menor o impacto ambiental tanto de EI como emissões de $\mathrm{CO}_{2}$. 
Figura 4 - Relação entre EI e IeC

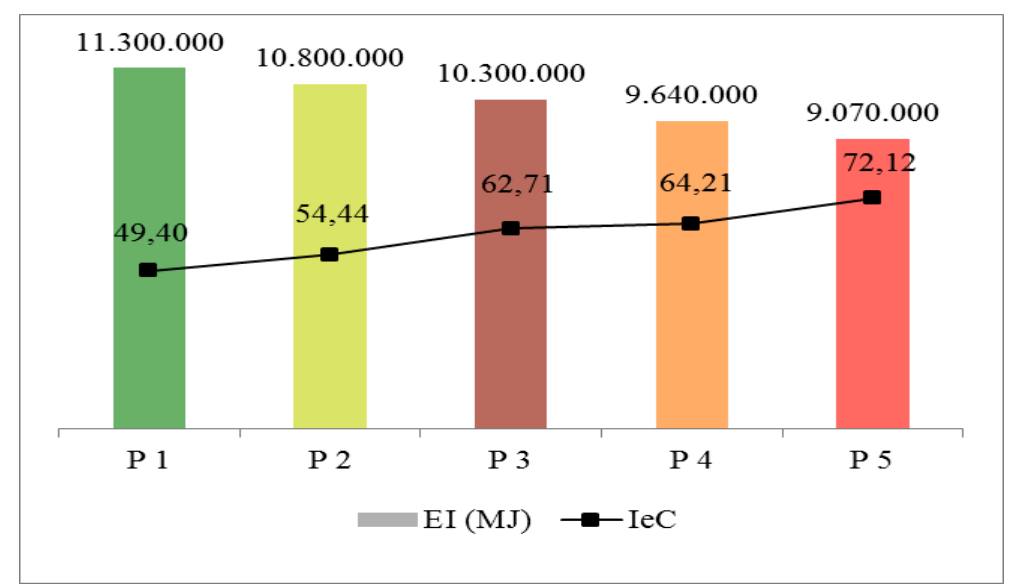

Figura 5 - Relação entre emissões de $\mathrm{CO}_{2}$ e IeC

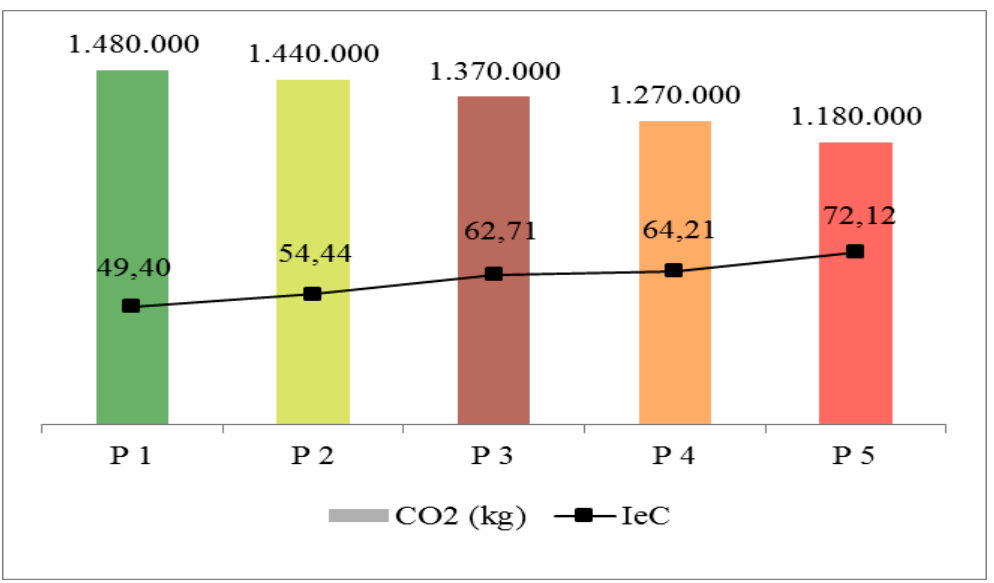

\section{CONCLUSÃO}

Entre os projetos analisados, comparando-se os que possuem respectivamente o menor e o maior IeC, de 49,4 (P1) e 72,12 (P5), obteve-se uma redução de 20\% quanto a EI do $\mathrm{P} 1$ para $\mathrm{P} 5$ e redução de $21 \%$ para emissões de $\mathrm{CO}_{2}$ em comparação a $\mathrm{P} 1$, considerandose a influência dos materiais e quantidade empregada dos mesmos. Desta forma, confirmando o pressuposto de que o aumento do $\mathrm{IeC}$ reduziria os impactos ambientais através dos quesitos analisados.

Salienta-se, que decisões de projeto com relação a forma do tipo arquitetônico, relacionadas a compacidade do projeto, causam reflexos diretamente relacionados a impactos ambientais, tanto quanto a energia incorporada quanto as emissões de $\mathrm{CO}_{2}$ dos materiais empregados no projeto.

Vale destacar que são necessárias maiores aprofundamentos na pesquisa bem como a ponderação de outros aspectos como o transporte dos materiais e investigação de outras técnicas construtivas, o que está em desenvolvimento na dissertação de mestrado já citada anteriormente. 


\section{AGRADECIMENTOS}

Ao Ministério da Ciência Tecnologia e Inovação, - MCTI, ao Conselho Nacional de Desenvolvimento Científico e Tecnológico - CNPq e ao Ministério das Cidades MCIDADES pelo fomento à pesquisa. À Caixa Econômica Federal e à empresa construtora participante, pela disponibilização dos projetos e orçamentos.

\section{REFERÊNCIAS}

AGOPYAN, V.; JOHN, V. M. O Desafio da Sustentabilidade na Construção Civil. [S.1.]: [s.n.], 2011. v. 5.).

BRANDÃO, D. Q. Avaliação da qualidade de arranjos espaciais de apartamentos baseada em aspectos morfo-topológicos e variáveis geométricas que influenciam na racionalização construtiva. Ambiente Construído, Porto Alegre, v. 6, n. 3, p. 53-67, 2006.

CAIXA ECONÔMICA FEDERAL (CEF). Cartilha Minha Casa Minha Vida. Brasília. Disponível em: $\quad<$ http://www.adh.pi.gov.br/minha_casa minha_vida.pdf $>$. Acesso em: 15 jun. 2013.

BRASIL. Eficiência energética em habitações de interesse social. Caderno 9. Brasilia: Ministério das Cidades/Ministério de Minas e Energia, 2005.

Balanço Energético Nacional. Ministério de Minas e Energia. Disponível em: <http://www.mme.gov.br/mme>. Acesso em: 11 março de 2014.

CARVALHO, M. T. M.; SPOSTO, R. M. Metodologia para avaliação da sustentabilidade de habitações de interesse social com foco no projeto. Ambiente Construído, Porto Alegre, v. 12, n. 1, p. 207-225, 2012.

DIAS, M. F. Modelo para estimar a geração de resíduos na produção de obras residenciais verticais. 2013. 168 f. Dissertação (Mestrado em Engenharia Civil) Programa de Pós-graduação em Engenharia Civil, Universidade do Vale do Rio dos Sinos (UNISINOS), São Leopoldo. 2013

GAUZIN-MÜLLER, D. Arquitectura ecológica. Barcelona: Gustavo Gili, 2002.

HEINECK, L. F. M.; FERNANDEZ, J. A. C. G. Modelo para avaliação qualitativa de projetos arquitetônicos, sob a ótica do usuário. Florianópolis, nov. 2004. Disponível em: $<\mathrm{http}: / / \mathrm{www}$.sindusconfpolis.org.br/artigosCientificos $>$.

LOBO, F. H. R. Inventário de emissão equivalente de dióxido de carbono e energia embutida na composição de serviços em obras públicas: Estudo de caso no Estado do Paraná. 2010. 212 f. Dissertação (Mestrado em Construção Civil) - Programa de PósGraduação em Construção Civil, Universidade Federal do Paraná, Curitiba. 2010.

LOBO, F. H. R.; SANTOS, A. P. L.; TAVARES, S. F. Revista Iberoamericana de Engenharia Industrial, Florianópolis, v. 2, n. 2, p. 26-43, 2010.

MASCARÓ, J. L. O Custo das Decisões Arquitetônicas. 5. ed. Porto Alegre: Masquatro, 2010.

MAYER, R. A gramática da habitação mínima : análise do projeto arquitetônico da habitação de interesse social em Porto Alegre e Região Metropolitana. 2012. 205 f. Tese (Doutorado em Arquitetura) - Programa de Pós-graduação em Arquitetura, Universidade Federal do Rio Grande do Sul, Porto Alegre, 2012. 
PAULSEN, J. S.; SPOSTO, R. M. A life cycle energy analysis of social housing in Brazil: Case study for the program "My House My Life". Energy and Buildings, n. 57, p. 95-102, 2013.

ROSSO, T. Aspectos geométricos do custo das edificações. Simpósio sobre barateamento da construção habitacional. Trabalho $n^{\circ}$ 83. Salvador, mar., 1978.

SCHNECK, E. R. Tipo arquitetônico em empreendimentos habitacionais de interesse social: impactos ambientais, diferenças no custo e em quesitos de habitabilidade. 2013. 168 f. Dissertação (Mestrado em Engenharia Civil) - Programa de Pós-graduação em Engenharia Civil, Universidade do Vale do Rio dos Sinos (UNISINOS), São Leopoldo. 2013

TAVARES, S. F. Metodologia de análise do ciclo de vida energético de edificações residenciais brasileiras. 2006. 225 f. Tese (Doutorado em Engenharia Civil) Programa de Pós-Graduação em Engenharia Civil, Universidade Federal de Santa Catarina (UFSC), Florianópolis. 2006.

WEINSTOCK, G. Agenda 21 para a Construção Sustentável. Relatório CIB Publicação 237, Novembro 2000. 